\title{
Equipment Operational Reliability Evaluation Method Based on RVM and PCA-Fused Features
}

\author{
Linbo Zhu ${ }^{D},{ }^{1}$ Dong Chen, ${ }^{2}$ and Pengfei Feng ${ }^{2}$ \\ ${ }^{1}$ School of Chemical Engineering and Technology, Xi'an Jiaotong University, Xi'an, China \\ ${ }^{2}$ Key Laboratory of Education Ministry for Modern Design and Rotor-Bearing System, Xi'an Jiaotong University, Xi'an, China
}

Correspondence should be addressed to Linbo Zhu; linbozhu@mail.xjtu.edu.cn

Received 1 December 2020; Revised 23 December 2020; Accepted 30 December 2020; Published 11 January 2021

Academic Editor: Yong Chen

Copyright (c) 2021 Linbo Zhu et al. This is an open access article distributed under the Creative Commons Attribution License, which permits unrestricted use, distribution, and reproduction in any medium, provided the original work is properly cited.

Reliability assessment is of great significance in ensuring the safety and reducing maintenance cost of equipment. The traditional statistical method is widely used to estimate the reliability of mass equipment; however, it cannot efficiently predict the overall reliability of single or small batch equipment due to lack of failure data. This paper introduced the operational reliability concept to describe the running condition of single or small batch equipment and proposed a method based on the combination of Relevance Vector Machines (RVMs) and Principal Component Analysis (PCA) to evaluate the operational reliability. Some representative characteristic indexes of operating equipment were firstly selected, and PCA was applied to obtain a hybrid index of the equipment's running condition. Then, a RVM prediction model was trained to predict the development of the hybrid index and corresponding probability density function (PDF). Based on this, the operational reliability of the equipment was calculated by the interval integral defined by the failure threshold and the predicted value of the hybrid index. The approach was validated using the experimental test conducted on the aero-engine rotor bearings. The results show a good agreement in the evaluations of the failure time between the proposed method and the experimental test.

\section{Introduction}

Reliability assessment is of great significance in ensuring the safety and reducing maintenance cost of equipment. In general, the reliability of equipment includes overall reliability and operational reliability. The former is obtained by statistical analysis of a large number of equipment failure data [1], and it reflects the overall reliability of the equipment throughout the lifetime. The latter is obtained from the performance degradation information of the equipment, and it reflects the real time reliability of the equipment [2]. In engineering field, single or small batch equipment is widely used, and failure data are very scarce in this case, so the traditional statistical method is not suitable for the reliability evaluation of this type of mechanical equipment, such as high precision NC machine tools, nuclear power facilities, aircraft [3-5], and so on. In contrast, the operational reliability is more of practical significance to ensure the safety of this type of equipment because it can be obtained in real time when the condition monitoring data are sampled continuously.

The operational reliability of the equipment is evaluated based on the performance degradation information, which is normally extracted from the monitoring data of the equipment [6]. The operating condition information obtained from the monitoring data is supposed to effectively reflect the evolution process of the dynamic operating characteristics of the equipment. In general, the equipment is considered to be failed or unreliable when some of its important performance parameters (such as vibration, noise, and so on) gradually reduced to the critical threshold [7, 8]; therefore, the relationship between performance parameters and operational reliability can be established.

There are three basic steps in evaluating the operational reliability of the equipment based on the performance degradation information. First, the characteristic index which reflects the operating condition is selected, and then the operational reliability calculation model based on the 
characteristic index is established. Third, the reliability of the equipment is predicted based on various prediction algorithms, such as artificial neural network [9], Support Vector Machines (SVMs) [10], Relevance Vector Machines (RVMs) [11], and so on. For the problem of characteristic index selection, the most related characteristic index to the health of the equipment is usually selected. For example, Casandra et al. [12] chosen the kurtosis as the bearing status indicators to evaluate the reliability of aero-engine rotor bearings. Shaban et al. [13] selected wear amount of tool as the performance degradation characteristic of cutting tools. $\mathrm{Zi}$ et al. [14] used the radial runout of the spindle end as the characteristic index of the electric spindle performance degradation. Li et al. [15] selected the waveform index of bearing vibration signal and used the energy obtained from wavelet packet decomposition as the degradation index of bearings to establish the reliability model. In the above references, only single characteristic index is used in operational reliability evaluation; this may result in the lack of robustness of the assessment method. Several references show that the reliability estimation accuracy can be improved by using multiple characteristic parameters. Widodo and Yang [11] introduced multiple parameters in reliability evaluation of aero-engine rotor bearings by using the Principal Component Analysis (PCA) method to fuse peak, kurtosis, and entropy to a hybrid index. Zheng et al. [16] also employed PCA to combine 10 variables, such as power system equipment availability coefficient, power supply reliability rate, capacity-to-load ratio, and so on, to estimate the reliability of the power supply system, and the reliability prediction accuracy was proved to be improved by adopting the hybrid index.

After selection of the characteristic index, the operational reliability can be obtained by several methods. One widely used method is to calculate the interval integral of the probability density function (PDF) of the selected index between the failure threshold and the observed value of the index [9]. In this method, the model of PDF of the selected index should be assumed and estimated firstly. Wang and Dragomir-Daescu [17] assumed that the PDF of wear data of the bearing in induction generators is a two-parameter Weibull distribution. Zi et al. [14] compared normal distribution, the logarithmic distribution, and the Weibull distribution in estimation the operational reliability of the spindle. Schömig and Infineon [18] obtained the fault data through simulation experiments and compared the accuracy of the reliability evaluation when the fault data obey the gamma distribution, exponential distribution, and Weibull distribution. The results show that the Weibull distribution is the best distribution in evaluation the operational reliability of semiconductor manufacturing equipment. It is easy to know that the accurate evaluation of PDF of the selected index is a critical issue in operational reliability estimation; however, the estimation accuracy of PDF is highly dependent on the number of samples.

Another commonly used method of operational reliability assessment is the K-M evaluator, in which the continued product of the ratio between the number of normal samples and the total number of samples is taken as the operational reliability of the equipment [19]. The main advantage of the K-M evaluator is that its computation process does not involve the estimation of PDF of samples, and the evaluation of operational reliability of equipment is simplified. Heng et al. [9] took the difference between the suspended data and CM (condition monitoring) data into consideration and combined the K-M and PDF methods to calculate the reliability of centrifugal pumps. He et al. [20] used the K-M estimator and the proportional hazards model to estimate the reliability of the engine exhaust valve based on mean air pressure, maximum coolant temperature, maximum fuel temperature, and other indexes. Fang et al. [21] used the K-M estimator to evaluate the reliability of the CNC honing hydraulic system based on pump output flow value. Based on tools wear amount, Chen et al. [22] estimated the reliability of CNC machine tools by combining Bayes and K-M estimators. However, the accuracy of the $\mathrm{K}-\mathrm{M}$ evaluator still depends on the number of observed failure samples, so it is also limited in applying in the single or small batch equipment.

In order to overcome the shortcomings of the traditional reliability evaluation methods, the RVM and PCA methods are introduced in this paper to develop a new evaluation method of the operational reliability for single or small patch equipment. RVM is a Bayesian-based machine learning method proposed by Tipping [23]; it is often adopted as a predictor in reliability prediction. For example, it is used in bearing reliability prediction [11], battery reliability prediction [24], and software reliability prediction [25]. In fact, RVM can also estimate the posterior probability of the predicted object at each prediction step. If it is applied to predict the characteristic index of equipment, the value of the characteristic index as well as its PDF can be obtained simultaneously. Based on the predicted PDF and the preset failure threshold, the operational reliability of the equipment can be obtained. At the same time, the PCA method is used to combine several characteristic indexes into one hybrid index to increase the robustness and the accuracy of the operational reliability prediction. The steps of method include the following. First, some representative characteristic indexes of running equipment are selected, and PCA is applied to these indexes to get the hybrid index. Then, the series of the hybrid index of long-term monitoring is used to train a single-step prediction RVM model to predict the value and probability density function (PDF) of the next step. Third, the operational reliability of the equipment is calculated by the interval integral defined by the failure threshold and the predicted value of the hybrid index.

In this method, only the performance degradation information of the object equipment is required, and there is no any other information from the same type equipment used, so the method is suitable for the reliability estimation problem of single equipment. The rest of the paper is organized as follows. Section 2 discusses RVM, PCA, and operational reliability calculation model and presents the framework of the methodology. Section 3 describes validation experiments, and Section 4 shows the results and discussion. Section 5 gives the conclusions. 


\section{Theoretical Method}

2.1. RVM Regression Model. Regression problem is defined as follows. Given an input $x_{i}(i=1,2, \ldots, N)$, by using the regression model, we can get its output $t_{i}(i=1,2, \ldots, N)$, that is, a set of sample set $\left\{x_{i}, t_{i}\right\}_{i=1}^{N}$ satisfies the following relationship:

$$
t_{i}=y\left(x_{i}, \boldsymbol{\omega}\right)+\varepsilon_{i},
$$

where $\varepsilon_{i}$ is the prediction noise of $x_{i}$ and is assumed to be zero-mean Gaussian distribution with variance $\sigma^{2}$. Moreover, $y\left(x_{i}, \boldsymbol{\omega}\right)$ can be expressed [26] as follows:

$$
y\left(x_{i}, \boldsymbol{\omega}\right)=\sum_{i=1}^{N} \omega_{i} K\left(x, x_{i}\right)+\omega_{0},
$$

where $\omega_{i}$ is an adjustable weight, $\omega_{0}$ is bias, and $K\left(x, x_{i}\right)$ is the corresponding kernel function; it is used to map the inputs $x_{i}$ from nonlinear space to high dimensional space and perform the linear regression in this space. The likelihood function of the training sample set is as follows:

$$
p\left(\mathbf{t} \mid \boldsymbol{\omega}, \sigma^{2}\right)=\left(2 \pi \sigma^{2}\right)^{-(N / 2)} \exp \left\{-\frac{1}{2 \sigma^{2}}\|\mathbf{t}-\boldsymbol{\Phi} \boldsymbol{\omega}\|^{2}\right\},
$$

where $\Phi=\left[\phi\left(x_{1}\right), \phi\left(x_{2}\right), \ldots, \phi\left(x_{N}\right)\right]^{T}$ is the $N \times(N+1)$ design matrix with $\phi\left(x_{i}\right)=\left[1, K\left(x_{i}, x_{1}\right), K\left(x_{i}, x_{2}\right), \ldots, K\left(x_{i}\right.\right.$, $\left.\left.x_{N}\right)\right]^{T}, x_{i}, i=1,2, \ldots, N$. In order to avoid the problem of over-learning, hyperparameter $\alpha_{i}$ is introduced to each weight coefficient $\omega_{i}$.

Supposing $\omega_{i}$ obeys a zero-mean Gaussian with variance $\alpha^{-1}$, then we can get the following:

$$
p(\boldsymbol{\omega} \mid \boldsymbol{\alpha})=\prod_{i=0}^{N} N\left(\omega_{i} \mid 0, \alpha_{i}^{-1}\right) .
$$

According to the Bayesian formula, we can get the posterior distribution function of the weight $\omega$ with the likelihood function equation (3) and a priori distribution function equation (4). That is,

$$
p\left(\boldsymbol{\omega} \mid \mathbf{t}, \boldsymbol{\alpha}, \sigma^{2}\right)=\frac{p\left(\mathbf{t} \mid \boldsymbol{\omega}, \sigma^{2}\right) p(\boldsymbol{\omega} \mid \boldsymbol{\alpha})}{p\left(\mathbf{t} \mid \boldsymbol{\alpha}, \sigma^{2}\right)}=N\left(\boldsymbol{\omega} \mid \mu, \sum\right),
$$

where the posterior covariance and mean are, respectively, as follows:

$$
\begin{aligned}
& \Sigma=\left(\sigma^{-2} \boldsymbol{\Phi}^{T} \boldsymbol{\Phi}+\mathbf{A}\right)^{-1}, \\
& \mu=\sigma^{-2} \sum \boldsymbol{\Phi}^{T} \mathbf{t} .
\end{aligned}
$$

In equation (6), $\mathbf{A}=\operatorname{diag}\left(\alpha_{1}, \alpha_{2}, \ldots, \alpha_{N}\right)$. The optimized hyperparameters $\alpha_{M P}$ and $\sigma_{M P}$ can be obtained by maximizing the marginal likelihood function $p\left(\mathbf{t} \mid \alpha, \sigma^{2}\right)$ with respect to $\alpha$ and $\sigma[11]$ :

$$
\max _{\alpha_{M P}, \sigma_{M P}} p\left(\mathbf{t} \mid \boldsymbol{\alpha}, \sigma^{2}\right)=\int p\left(\mathbf{t} \mid \boldsymbol{\omega}, \sigma^{2}\right) p(\boldsymbol{\omega} \mid \boldsymbol{\alpha}) d \boldsymbol{\omega}=(2 \pi)^{-(N / 2)}\left|\sigma^{2} \mathrm{I}+\boldsymbol{\Phi} \mathbf{A}^{-1} \Phi^{T}\right|^{-(1 / 2)} \exp \left\{-\frac{1}{2} t^{T}\left(\sigma^{2} \mathrm{I}+\boldsymbol{\Phi} \mathbf{A}^{-1} \Phi^{T}\right)^{-1} \mathbf{t}\right\}
$$

Given a new input value $x_{*}$, the target output is as follows:

$$
p\left(t_{*} \mid x_{*}, \alpha_{M P}, \sigma_{M P}^{2}\right)=\int p\left(t_{*} \mid \boldsymbol{\omega}, \sigma_{M P}^{2}\right) p\left(\boldsymbol{\omega} \mid \mathbf{t}, \alpha_{M P}, \sigma_{M P}^{2}\right) d \boldsymbol{\omega} .
$$

which can be easily computed since both integrated terms obeys Gaussian distribution, that is,

$$
p\left(t_{*} \mid x_{*}, \alpha_{M P}, \sigma_{M P}^{2}\right)=N\left(t_{*} \mid y_{*}, \sigma_{*}^{2}\right),
$$

where the mean and variance are, respectively, as follows:

$$
\begin{aligned}
& y_{*}=\mu^{T} \phi\left(x_{*}\right), \\
& \sigma_{*}^{2}=\sigma_{M P}^{2}+\phi\left(x_{*}\right)^{T} \sum \Phi\left(x_{*}\right) .
\end{aligned}
$$

Therefore, the RVM learning method based on the Bayesian framework can be used to predict the probability and obtain the forecast value and its probability distribution. If the input and output data are from a time series (for example, precipitation data), RVM can be used to predict the future value of the time series.
2.2. Operational Reliability Calculation Model. The traditional reliability theory can only provide the overall reliability assessment for mass equipment, but in engineering field, single or small batch equipment is widely used and failure data are very scarce in this case; people are more concerned about the reliability of the particular equipment in operation. To solve the reliability evaluation problem of single or small batch equipment, Heng et al. [9] proposed an operational reliability model based on the equipment running characteristic index, and the model is described as follows.

Let $Y_{i}(t)$ be the value of the condition characteristic index for equipment $i$ at operating age $t$, and $\mathbf{Y}(t)=\left[Y_{1}(t), Y_{2}(t), \ldots, Y_{m}(t)\right]^{T}$ containing the condition values from all of the $m$ historical equipment in interval $t$, and $Y_{\text {thresh }}$ be the threshold of the characteristic index; if $Y_{i}(t)>Y_{\text {thresh }}$, the equipment is considered to be failed. $f(Y \mid t)$ is the corresponding PDF of $\mathbf{Y}(t)$; the overall reliability at time $t$ is defined as follows:

$$
R(t)=P\left(Y(t)<Y_{\text {thresh }}\right)=\int_{0}^{Y_{\text {thresh }}} f(Y \mid t) d Y .
$$


The operational reliability of equipment $i$ at time $t+k \Delta t$ is defined as follows:

$$
R_{i}(t+k \Delta t)=\prod_{j=1}^{k} \frac{P\left[y_{\text {thresh }}>Y_{i}(t+j \Delta) \geq y_{i, t+j \Delta} \mid y_{\text {thresh }}>Y_{i}(t+(j-1) \Delta) \geq y_{i, t+(j-1) \Delta}, \ldots\right]}{P\left[Y_{i}(t+j \Delta) \geq y_{i, t+j \Delta} \mid y_{\text {thresh }}>Y_{i}(t+(j-1) \Delta) \geq y_{i, t+(j-1) \Delta}, \ldots\right]}=\prod_{j=1}^{k} \frac{\int_{y_{i, t+j \Delta}}^{y_{\text {thres }}} f(y \mid t+j \Delta) d y}{\int_{y_{i, t+j \Delta}}^{\infty} f(y \mid t+j \Delta) d y}
$$

where $\int_{y_{i, t+j \Delta}}^{y_{\text {thresh }}} f(y \mid t+j \Delta) d y$ is the integral of the PDF between the observed degradation index of device $i$ and the threshold and $\int_{y_{i, t+j \Delta}}^{\infty} f(y \mid t+j \Delta) d y$ is the integral of the PDF over all possible values equal to or higher than the observed degradation index of device $i$.

In summary, if the value of the characteristic index and the corresponding PDF can be obtained, the operational reliability of equipment can be calculated by equation (12).

2.3. $P C A$. In the process of reliability analysis, several vibration characteristics of the equipment can be obtained simultaneously. It will be too complicated to evaluate the reliability if we choose all of them. To improve the stability of reliability estimation, an intuitive approach is to fuse all characteristics to conduct the reliability analysis [27]. In this paper, the PCA method is adopted to fulfill this requirement. PCA is a statistical procedure that uses an orthogonal transformation to convert a set of observations of possibly correlated variables into a set of values of linearly uncorrelated variables called principal components. If the vibration characteristics have similar trends with respect to time, the first principal component will contain the most information of all characteristics and can be used as the reliability index.

The principal component analysis method is obtained by projecting the original vector as a new coordinate space consisting of the eigenvectors of the covariance matrix of the original variables $[28,29]$. For a given vibration characteristic vector set of $m$ features $\mathbf{X}=\left\{x_{1}, \cdots, x_{m}\right\}, x_{i} \in R^{n}$ which consists of feature vectors, the covariance matrix $C$ is given as equation (13), in which $\mu$ is mean value of $x_{i}$ :

$$
C=\frac{1}{m} \sum_{i=1}^{m}\left(x_{i}-\mu\right)\left(x_{i}-\mu\right)^{T}
$$

Then, the eigenvalues $\lambda_{i}(i=1,2, \ldots, n)$ and corresponding eigenvectors $v_{i}(i=1,2, \ldots, n)$ of $C$ are given as follows:

$$
C v_{i}=\lambda_{i} \nu_{i}
$$

Sort the eigenvalues in decreasing order $\lambda_{1} \geq \lambda_{2} \geq \ldots$, $\geq \lambda_{n}$, and composite the first $k$ eigenvalues $\Delta=\left(\lambda_{1}, \lambda_{2}, \ldots, \lambda_{k}\right)$ and the corresponding eigenvectors $\mathbf{V}=\left(v_{1}, v_{2}, \ldots, v_{k}\right)$ and then transform the original data $\mathbf{X}$ onto the new subspace $\mathbf{V}$ and get the transformed data $\mathbf{Y}$ :

$$
\mathbf{Y}=\mathbf{V}^{T} \mathbf{X}
$$

The number of principle component is selected depending on the cumulative contribution $R_{k}$, which is usually set more than $85 \%-90 \%$ :

$$
R_{k}=\frac{\sum_{i=1}^{k} \lambda_{i}}{\sum_{i=1}^{n} \lambda_{i}}
$$

2.4. Operational Reliability Prediction Methodology. As described above, the method employs performance degradation data of equipment which are obtained from the continuously monitoring of equipment's running condition. Some representative characteristic indexes of equipment, which can describe the degradation process of equipment from normal to failure, are selected. PCA is applied to deal with these characteristic indexes to obtain the hybrid index. The RVM regression model is then trained to obtain the hybrid index. Based on the RVM regression method, the posterior distribution function and the predicted value of the characteristic index in different time can be obtained. Finally, the operational reliability can be calculated by the mentioned operational reliability calculation model (Section 2.2). The total process is illustrated in Figure 1.

\section{Experimental Investigation}

The data of fatigue life experiments of aero-engine rotor bearings [30] are adopted to validate the proposed method. In experiments, four bearings were installed on a shaft. The rotation speed was kept constant at $2000 \mathrm{rpm}$, radial load of $6000 \mathrm{lbs}$ is applied onto the shaft, and all bearings were force lubricated. Three different types failure are tested. The first failure type is inner ring failure; it comes from the $3 \#$ bearing (it is named A bearing in the paper). The second failure type is composite failure of outer ring and rolling element; it comes from the of 4 \# bearing (named B bearing), and the third failure is outer ring spalling of 1 \# bearing (named $\mathrm{C}$ bearing). The data were collected every 20 minutes, and the sampling frequency is $12 \mathrm{kHz}$ in each measurement cycle. The measurement time is 43000 minutes, and the data length is 2150 points. It means that the measurement time is 20 times the measurement points. The selected data in the paper come from reference [30], but they do not include the one of the first 5 days considering the instability of the previous data.

Several time-domain characteristic indexes of bearing A are shown in Figure 2. From RMS and waveform curves, it can be observed that at the beginning of experiments, the curves keep stable (the small change at the very beginning is considered to be caused by the instability of the running stage), and the main fluctuations are happened near to 1800 measurement points that means the early failure is happed in the bearing. This trend can also be found in kurtosis and peak index. While for mean value, it can be observed that its 


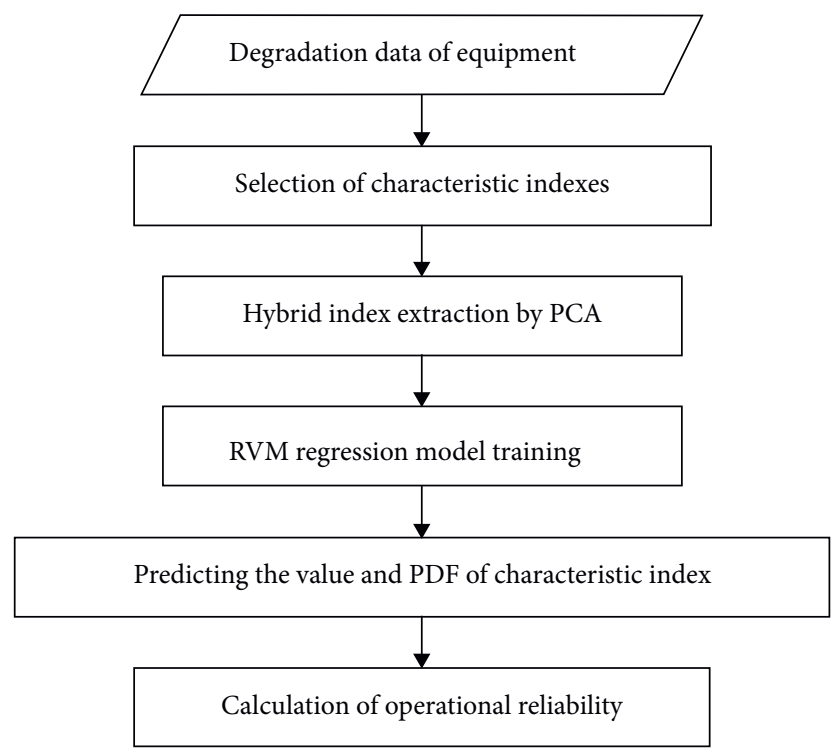

FigURE 1: Operational reliability prediction process.

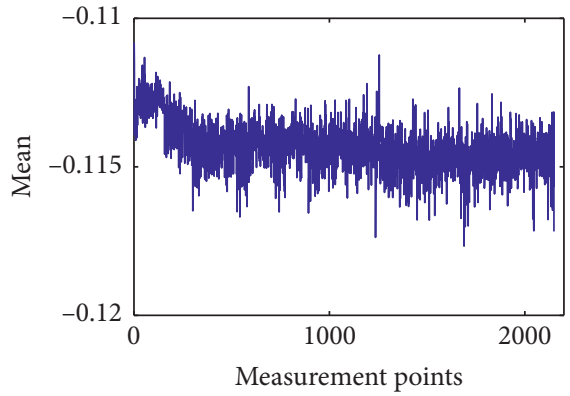

(a)

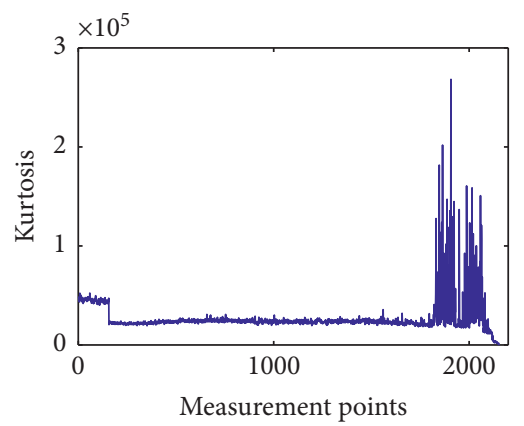

(d)

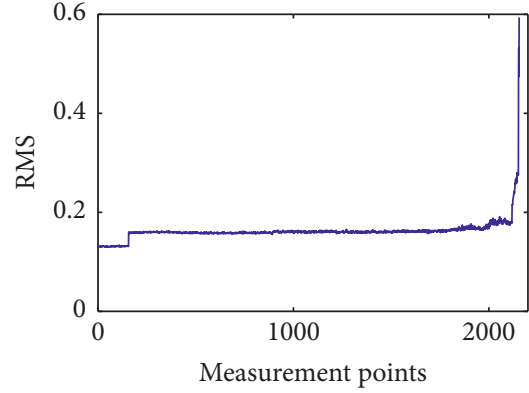

(b)

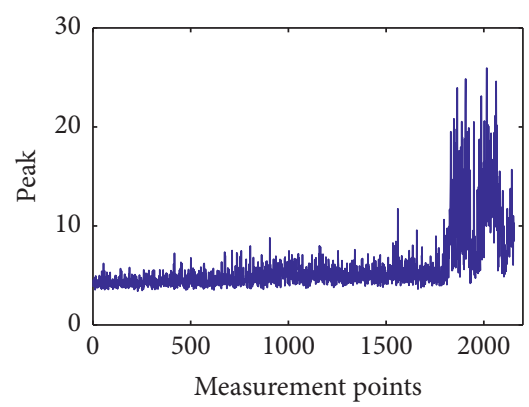

(e)

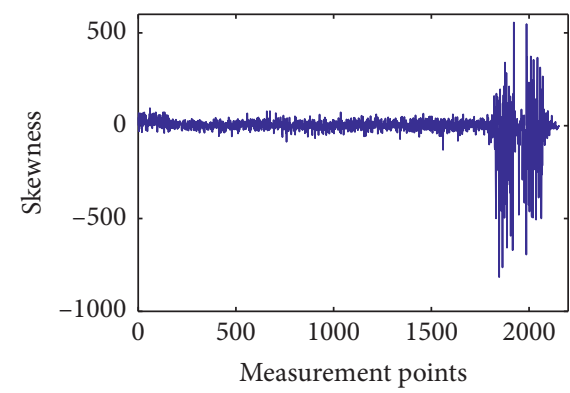

(c)

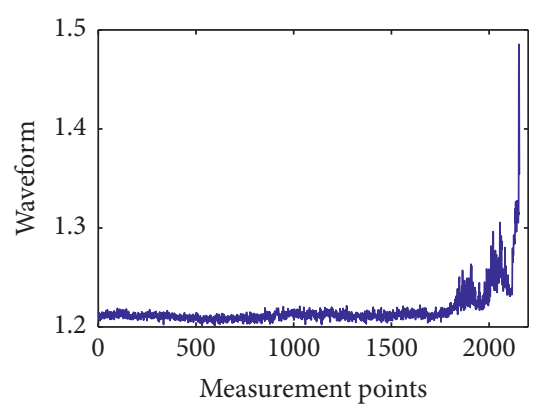

(f)

Figure 2: Vibration signal time-domain indexes: (a) mean, (b) RMS, (c) skewness, (d) kurtosis, (e) peak, and (f) waveform.

value decreases with the running of experiment and is not sensitive to the failure. The skewness index oscillates sharply when the early failure happens, which does not conform to our expectation of continuous increase or continuous reduction of the selected index in operational reliability evaluation.

From Figure 2, it is also can be found that different characteristic indexes have different sensitivity to the failure. Though the RMS index and waveform index rise sharply with the increase in the severity of the failure, the changes on the trend of the curves caused by early failure are relatively small compared with the peak index and kurtosis index. As for the kurtosis index, it increases at the early stage of failure, while decreases with the development of the failure. To make the characteristic index both sensitive and robust to the failure, RMS, waveform index, and peak value are selected and combined with the PCA method, and the obtained hybrid index of bearing A, B, and C is shown in Figure 3 . 


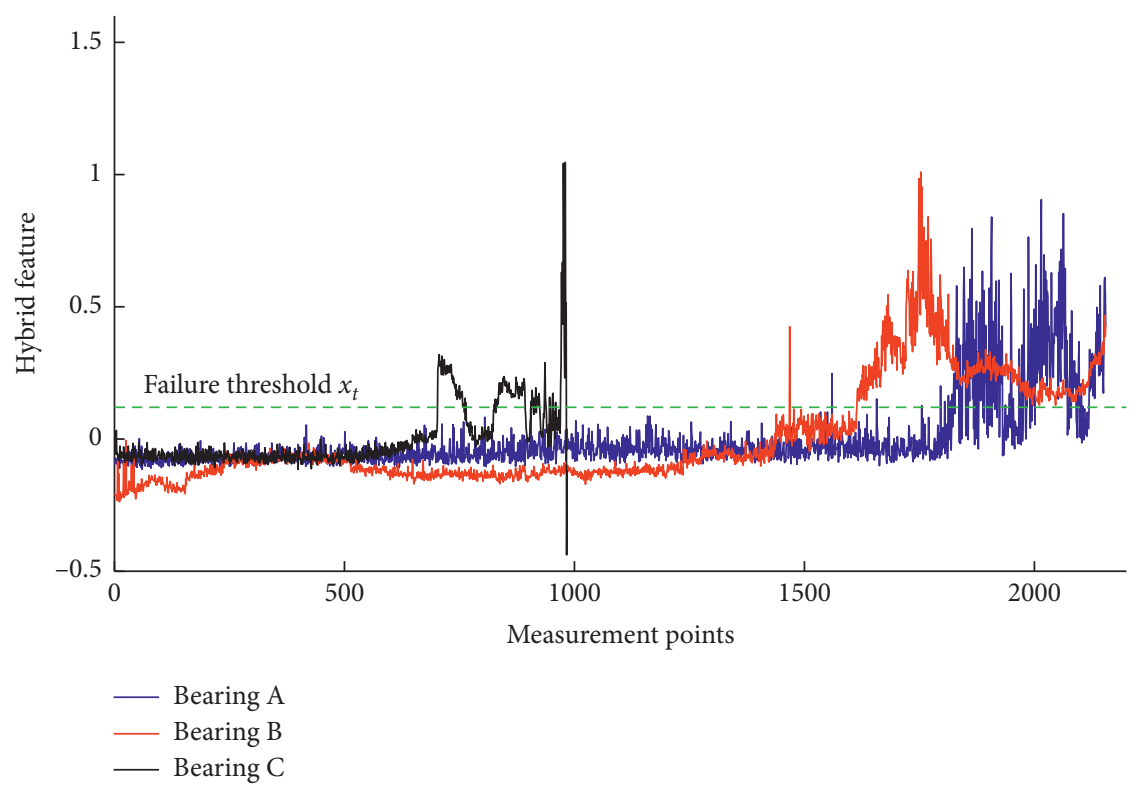

Figure 3: Hybrid index of three bearing.

An empirical threshold $x_{t}=0.12$ is assumed and plotted in Figure 3 with dotted line. It can be observed that when the hybrid index is smaller than the threshold, the hybrid index changes more smoothly, and the bearing is supposed to work in normal condition. When the threshold is exceeded, the hybrid index increases rapidly and has a relatively violent fluctuation, which means the happening of failure in the bearing. The failure time of the three bearings determined by the failure threshold is 703,1614 , and 1821 points, respectively.

\section{Results and Discussion}

The hybrid index of the first 1500 points of bearing $\mathrm{A}$, the first 1500 points of bearing $\mathrm{B}$, and the first 650 points of bearing $C$ are selected, respectively, to train the single-step RVM prediction model for each bearing. The embedding dimension is set to 20 , the RVM kernel parameter optimization range is $[0.1,20]$, and the optimization objective fitness function is as follows [31]:

$$
\text { fitness }=\frac{\left(\mathrm{RMS}_{\text {train }} * n_{1}+\mathrm{RMS}_{\text {test }} * n_{2}\right) * \mathrm{RV}}{n_{1}+n_{2}},
$$

where $\mathrm{RMS}_{\text {train }}$ and $\mathrm{RMS}_{\text {test }}$ are training errors and the test errors and are obtained with the 5-folds cross-validation method, respectively. $n_{1}$ and $n_{2}$ are the number of training samples and the number of test samples, and RV is the number of relevance vectors. Minimizing this fitness function makes the trade-off of kernel parameter between training and test errors and makes the trained model to have the best prediction accuracy. The obtained optimized kernel parameters for each model are given in Table 1, and the prediction accuracy is defined as follows:

$$
\text { accuracy }=\left(1-\frac{\left|t_{a}-t_{p}\right|}{t_{a}}\right) \times 100 \% \text {, }
$$

TABLE 1: Optimization results of kernel parameters for the RVM model.

\begin{tabular}{lcc}
\hline RVM model & Kernel parameter & Fitness \\
\hline Bearing A & 16.8 & 0.0334 \\
Bearing B & 6.2 & 0.07 \\
Bearing C & 9.9 & 0.0203 \\
\hline
\end{tabular}

where $t_{a}$ is the actual failure time and $t_{p}$ is the predicted failure time. It must be indicated that the failure time is 20 times the failure point for this case.

The prediction results on the hybrid index of three bearings are shown in Figure 4(a), Figure 5(a), and Figure 6(a), respectively. In each graph, the red points represent actual data and the blue points represent the predicted data. The results show that the change trend of the predicted value closely matches the actual value; it means RVM has acceptable accuracy in predicting the time series of the hybrid index. Figure 4(b), Figure 5(b), and Figure 6(b), respectively, show the prediction results on operational reliability of three bearings and corresponding enlarged diagram. It can be observed that at the early stage of bearing total lifetime, the bearings have relatively high operational reliability and relatively small degradation trend (from the start of operation to approximate 1823, 1512, and 704 measurement points of three bearings, respectively). After that, the operational reliabilities of three bearings show sharp drops, which means the initiation of a defect. At 1836 points of bearing $A, 1623$ points of bearing $B$, and 714 points of bearing $\mathrm{C}$, the operational reliability is forecasted to fall close to zero, meaning that the bearings are closed to complete failure. This degradation process indicates that the occurrence and development of bearing fault is a rapid process and will happen in very short time, compared with the total service life of the bearing. 


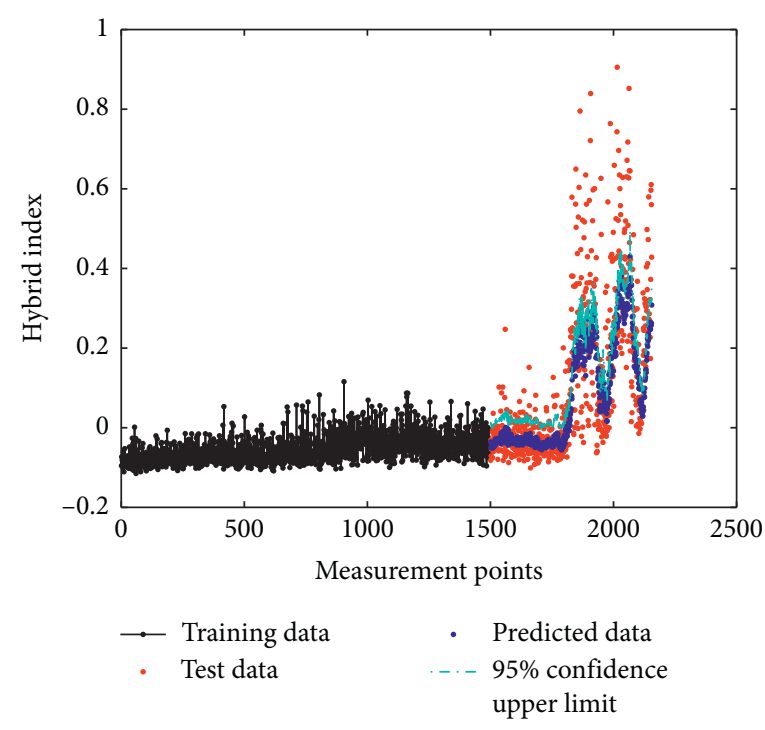

(a)

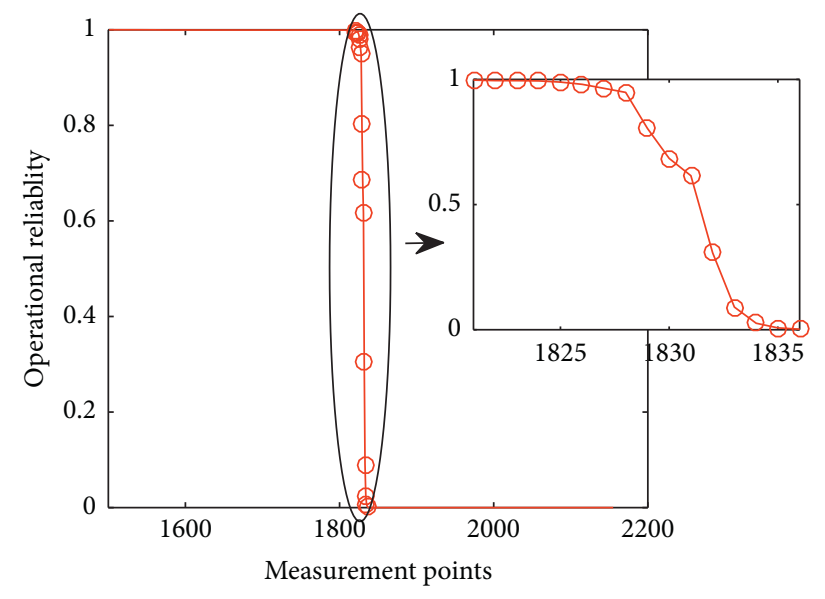

(b)

FIgURE 4: Prediction results of bearing A: (a) hybrid index and (b) operational reliability.

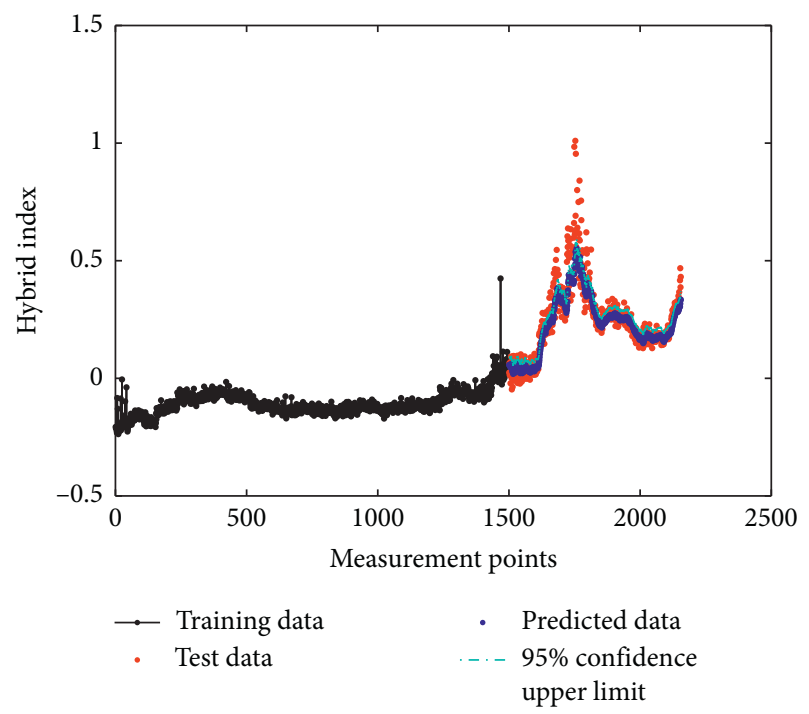

(a)

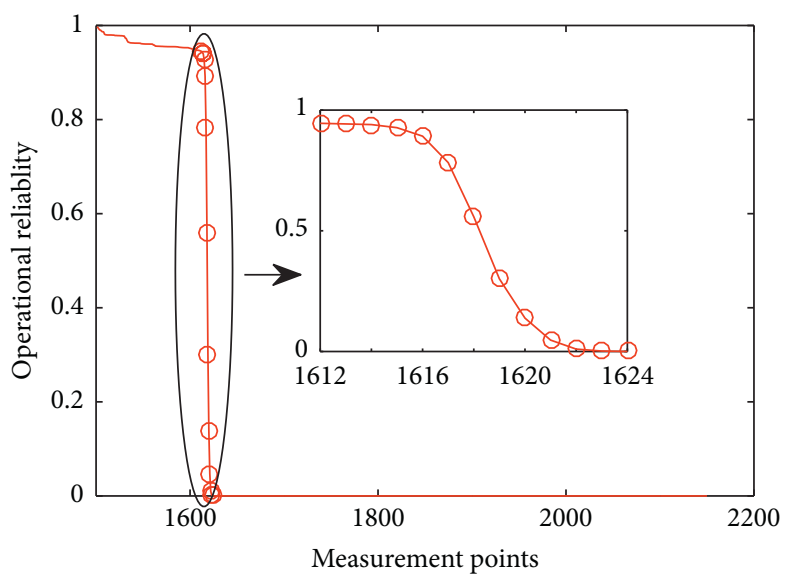

(b)

FIgURe 5: Prediction results of bearing B: (a) hybrid index and (b) operational reliability.

It is also noted that three bearings have experienced different time from initiation of the defect to final failure because of the difference of bearing fault. In fact, this failure mechanism and fault development process can also be observed in Figure 3, in which the similar evolutionary process of the hybrid index is shown; it proves that the RVM prediction models have learned the failure pattern of three bearings. The prediction accuracy obtained from the reliability curve with equation (18) is shown in Table 2. The results show that the prediction accuracy on failure time of three bearings is all above $98 \%$. The results suggest that the operational reliability evaluation model captures the nonlinear relationship between the hybrid index and the actual health state of the monitored equipment.

In addition, in order to verify the validity of the hybrid index, this paper also compares the reliability evaluation results based on the single index with that on the hybrid index. Table 3 gives the results on bearing $\mathrm{C}$ as an example; it was shown that the prediction accuracy of the failure time obtained with the hybrid index is higher than that obtained with the single index, indicating that the hybrid index can synthetically consider a variety of information and obtain better evaluation results. 


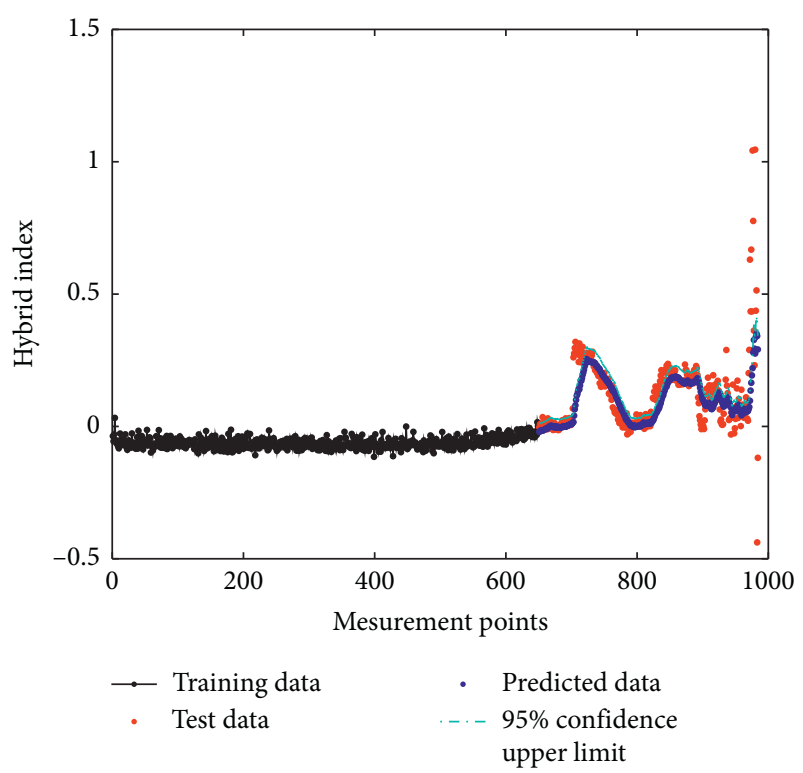

(a)

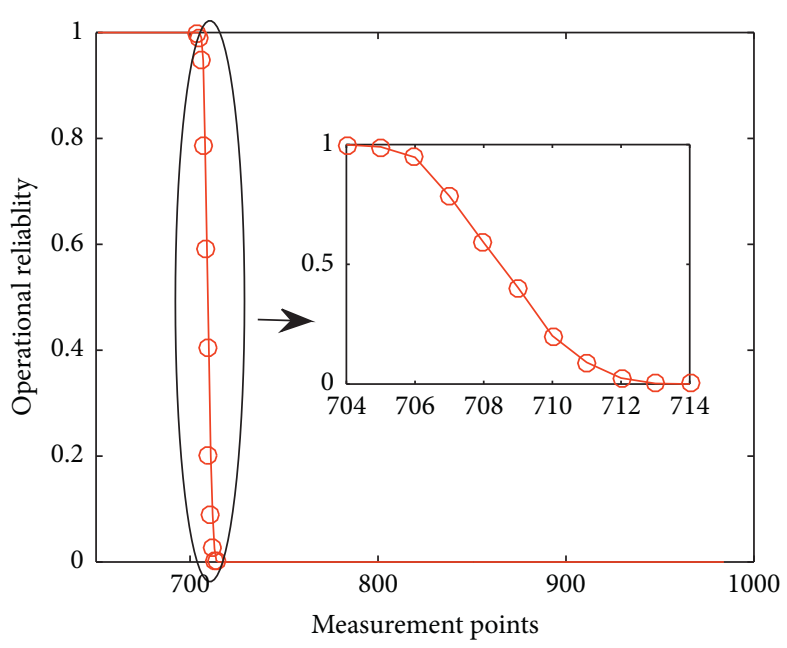

(b)

Figure 6: Prediction results of bearing C: (a) hybrid index and (b) operational reliability.

TABLE 2: Three bearing failure time prediction results.

\begin{tabular}{lccc}
\hline Bearing & Actual failure time $(\mathrm{min})$ & Predicted failure time $(\mathrm{min})$ & Accuracy $(\%)$ \\
\hline A & 36420 & 36720 & 99.2 \\
B & 32280 & 32460 & 99.4 \\
C & 14060 & 14280 & 98.4 \\
\hline
\end{tabular}

TABLe 3: Single index method and hybrid index method prediction results of bearing C.

\begin{tabular}{lccc}
\hline Characteristic index & Actual failure time $(\mathrm{min})$ & Predicted failure time $(\mathrm{min})$ & Accuracy $(\%)$ \\
\hline Waveform & 14060 & 14380 & 97.7 \\
Peak & 14080 & 14360 & 98.0 \\
Kurtosis & 14080 & 14480 & 97.2 \\
Hybrid index & 14060 & 14280 & 98.4 \\
\hline
\end{tabular}

\section{Conclusions}

This paper presents a method to evaluate the operational reliability for single or small patch equipment based on RVM and PCA. The PCA was used to fuse the features and obtain the hybrid index which can represent the degradation information of the equipment more robustly. The RVM was used to establish a single-step prediction model of the hybrid index and predict the future value of the hybrid index and the corresponding PDF at a certain moment. Based on PDF and the predicted value of the hybrid index, the operational reliability of the equipment is obtained with the interval integral defined by the failure threshold and the predicted value of the hybrid index. The performance of the proposed method is validated by predicting the failure time of aero-engine rotor bearings. This paper also compares the reliability evaluation results based on the hybrid index with the reliability evaluation results based on a single index. The results proves the plausibility and effectiveness of the proposed method.

\section{Data Availability}

The data used to support the findings of this study are included within the article.

\section{Conflicts of Interest}

The authors declare that they have no conflicts of interest.

\section{Acknowledgments}

This study was supported by the National Natural Science Foundation of China (nos. 51805412 and 51635010), Science and Technology Major Project of Henan Province (no. 191110213300), National Key Research and Development Project (no. 2020YFB2007900), and China Postdoctoral Science Foundation (nos. 2018M631144 and 2019T120897). 


\section{References}

[1] P. D. T. O'Connor, "Commentary: reliability-past, present, and future," IEEE Transactions on Reliability, vol. 49, no. 4, pp. 335-341, 2000.

[2] Z. He, H. Cao, Y. Zi, and B. Li, "Developments and thoughts on operational reliability assessment of mechanical equipment," Journal of Mechanical Engineering, vol. 50, no. 2, pp. 171-186, 2014.

[3] T.-H. Fan and C.-C. Chang, "A Bayesian zero-failure reliability demonstration test of high quality electro-explosive devices," Quality and Reliability Engineering International, vol. 25, no. 8, pp. 913-920, 2009.

[4] M. Balesdent, J. Morio, and J. Marzat, "Recommendations for the tuning of rare event probability estimators," Reliability Engineering \& System Safety, vol. 133, pp. 68-78, 2015.

[5] H. Li, F. Chen, Z. Yang, Y. Kan, and L. Wang, Bayesian Reliability Assessment Method for Single NC Machine Tool under Zero Failures, pp. 291-302, Springer, Berlin, Heidelberg, 2015.

[6] J. C. Ferreira, M. A. Freitas, and E. A. Colosimo, "Degradation data analysis for samples under unequal operating conditions: a case study on train wheels," Journal of Applied Statistics, vol. 39, no. 12, pp. 2721-2739, 2012.

[7] D. Xu and W. Zhao, "Reliability prediction using multivariate degradation data," in Proceedings of Conference on Reliability and Maintainability Symposium, pp. 337-341, Alexandria, VA, USA, January 2005.

[8] M. S. Chang, Y. I. Kwon, and B. S. Kang, "Design of reliability qualification test for pneumatic cylinders based on performance degradation data," Journal of Mechanical Science and Technology, vol. 28, no. 12, pp. 4939-4945, 2014.

[9] A. Heng, A. C. C. Tan, J. Mathew, N. Montgomery, D. Banjevic, and A. K. S. Jardine, "Intelligent condition-based prediction of machinery reliability," Mechanical Systems and Signal Processing, vol. 23, no. 5, pp. 1600-1614, 2009.

[10] L. Chato, S. Tayeb, and S. Latifi, "A genetic algorithm to optimize the adaptive support vector regression model for forecasting the reliability of diesel engine systems," in Proceedings of IEEE Conference on Computing and Communication Workshop and Conference, pp. 1-7, Las Vegas, NV, USA, January 2017.

[11] A. Widodo and B.-S. Yang, "Application of relevance vector machine and survival probability to machine degradation assessment," Expert Systems with Applications, vol. 38, no. 3, pp. 2592-2599, 2011.

[12] W. Caesarendra, A. Widodo, and B.-S. Yang, "Application of relevance vector machine and logistic regression for machine degradation assessment," Mechanical Systems and Signal Processing, vol. 24, no. 4, pp. 1161-1171, 2010.

[13] Y. Shaban, S. Yacout, and M. Aly, "Condition-based reliability prediction based on logical analysis of survival data," in Proceedings of IEEE Conference on Reliability and Maintainability Symposium, pp. 1-6, Orlando, FL, USA, January 2017.

[14] J. Zi, H. Liu, X. Jiang, and L. Liu, "Reliability assessment of electric spindle based on degradation values distribution," China Mechanical Engineering, vol. 25, pp. 807-812, 2014.

[15] H.-K. Li, Z.-X. Zhang, X.-G. Li, and Y.-J. Ren, "Reliability prediction method based on state space model for rolling element bearing," Journal of Shanghai Jiaotong University (Science), vol. 20, no. 3, pp. 317-321, 2015.

[16] Y. Zheng, G. Sun, Z. Wei, F. Zhao, and Y. Sun, "A novel power system reliability predicting model based on PCA and RVM,"
Mathematical Problems in Engineering, vol. 2013, Article ID 648250, , 2013.

[17] W. Wang and D. Dragomir-Daescu, "Reliability quantification of induction motors-accelerated degradation testing approach," in Proceedings of IEEE Conference on Reliability and Maintainability Symposium, pp. 325-331, Seattle, WA, USA, February 2002.

[18] A. K. Schömig and Infineon, On the Suitability of the Weibull Distribution for the Approximation of Machine Failures, in Proceedings of IIE Annual Conference, pp. 1-7, Oregon, Portland, USA, May 2003.

[19] E. L. Kaplan and P. Meier, "Nonparametric estimation from incomplete observations," Journal of the American Statistical Association, vol. 53, no. 282, pp. 457-481, 1958.

[20] Y. He, A. Kusiak, T. Ouyang, and W. Teng, "Data-driven modeling of truck engine exhaust valve failures: a case study," Journal of Mechanical Science and Technology, vol. 31, no. 6, pp. 2747-2757, 2017.

[21] M. Fang, G. Zhou, Y. Cheng, and X. Lei, "Reliability prediction method for hydraulic system of CNC honing machine based on running status information," Applied Science and Technology, vol. 39, no. 6, pp. 30-33, 2012.

[22] B. Chen, X. Chen, Z. He, and B. Li, "Operating condition information-based reliability prediction of cutting tool," Journal of Xi'an Jiaotong University, vol. 44, no. 9, pp. 74-77, 2010.

[23] M. E. Tipping, "Sparse bayesian learning and the relevance vector machine," Journal of Machine Learning Research, vol. 1, pp. 211-244, 2001.

[24] H. Li, D. Pan, and C. L. P. Chen, "Intelligent prognostics for battery health monitoring using the mean entropy and relevance vector machine," IEEE Transactions on Systems, Man, and Cybernetics: Systems, vol. 44, no. 7, pp. 851-862, 2014.

[25] J. Lou, Y. Jiang, Q. Shen, Z. Shen, Z. Wang, and R. Wang, "Software reliability prediction via relevance vector regression," Neurocomputing, vol. 186, pp. 66-73, 2016.

[26] R. Mohebian, M. A. Riahi, and M. Afjeh, "Detection of the gas-bearing zone in a carbonate reservoir using multi-class relevance vector machines (RVM): comparison of its performance with SVM and PNN," Carbonates and Evaporites, vol. 33, no. 2-4, pp. 347-357, 2018.

[27] L. Cui, N. Wu, W. Wang, and C. Kang, "Sensor-based vibration signal feature extraction using an improved composite dictionary matching pursuit algorithm," Sensors, vol. 14, no. 9, pp. 16715-16739, 2014.

[28] F. Wang, J. Sun, D. Yan, S. Zhang, L. Cui, and Y. Xu, "A feature extraction method for fault classification of rolling bearing based on PCA," Journal of Physics: Conference Series, vol. $628,2015$.

[29] I. T. Jolliffe and J. Cadima, "Principal component analysis: a review and recent developments," Philosophical Transactions of the Royal Society A: Mathematical, Physical and Engineering Sciences, vol. 374, no. 2065, 2016.

[30] H. Qiu, J. Lee, J. Lin, and G. Yu, "Wavelet filter-based weak signature detection method and its application on rolling element bearing prognostics," Journal of Sound and Vibration, vol. 289, no. 4-5, pp. 1066-1090, 2006.

[31] G. Li, G. Wang, and H. Xue, "Optimizing method to kernel function parameters of RVM," Control Engineering of China, vol. 17, pp. 335-337, 2010. 